\title{
The effect of lacosamide on bone tissue in orchidectomised male albino Wistar rats
}

\author{
Julius Simko ${ }^{a}$, Sona Fekete ${ }^{b}$, Jana Malakova ${ }^{b}$, Jan Kremlacekc, Jiri Horacek ${ }^{d}$, Helena Zivna ${ }^{e}$, Vladimir Palicka ${ }^{b}$, Pavel Zivny ${ }^{b}$
}

Aims. While most antiepileptic drugs (AEDs) have been associated with various adverse effects on bone health, for the recently introduced lacosamide (LCM) no corresponding data have been published. The present study evaluates the effect of LCM on bone mineral density, bone turnover markers, and bone mechanical strength in a rat model.

Methods. 16 orchidectomized Wistar rats were divided into control and experimental groups, 8 rats each. Dual energy X-ray absorptiometry was used to measure bone mineral density (BMD). As bone metabolism markers, the concentrations of bone markers were assayed in bone homogenate. In addition, both femurs were measured and used for biomechanical testing.

Results. Compared to the control group, we found lower BMD in the experimental group in the area of the left (8\%) as well as the right femur (12\%), all differences being statistically significant. In both femur diaphyses, but not in lumbar vertebrae, BMD was lower in the LCM group, suggesting a preferential effect on cortical bone. However, neither the thickness of the diaphyseal cortical bone nor the fragility in biomechanical testing was different between the groups. Of the bone metabolism markers, the significant decline was in procollagen type I N-terminal peptide (PINP) levels (37.4\%), suggesting a decrease in osteoid synthesis.

Conclusion: We assume then that long-lasting exposure to LCM can represent a certain risk to the health of bone in the setting of gonadal insufficiency. Further studies will be needed to confirm these findings and to determine how high the risk will be in comparison to the other AEDs.

Key words: antiepileptic drugs, bone markers, bone mineral density, bone turnover, biomechanical strength

Received: July 8, 2013; Accepted with revison: January 16, 2014; Available online: January 27, 2014 http://dx.doi.org/10.5507/bp.2014.006

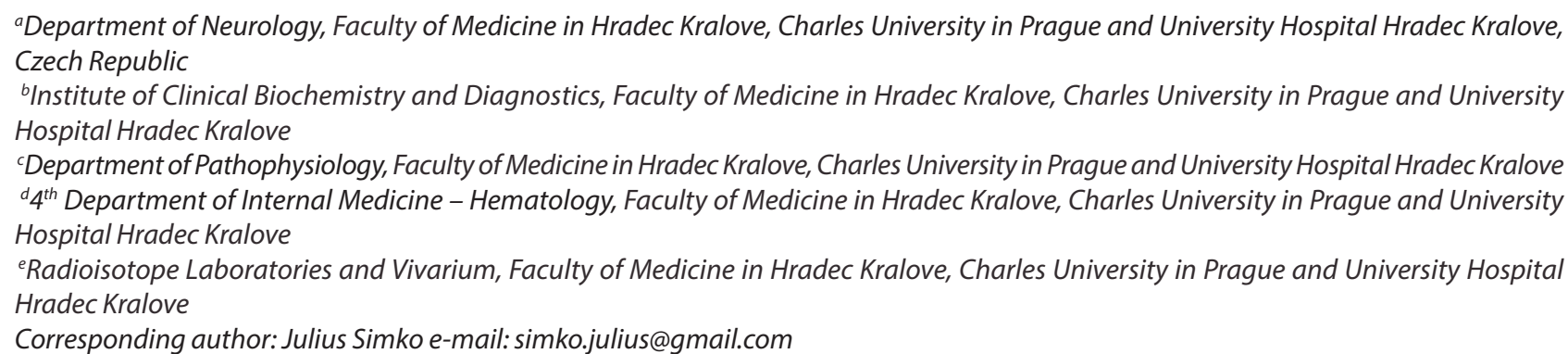

\section{INTRODUCTION}

Their use in epilepsy and the treatment of pain, and wide therapeutic use in psychiatry make antiepileptic drugs (AEDs) a pharmaco-epidemiologically important group of drugs both in the adult as well as the juvenile population ${ }^{1-3}$. Osteopathies occurring with long-term chronic antiepileptic treatment were first noted in the late sixties ${ }^{4,5}$. Since that time a number of theories have been proposed to explain why AEDs affect bone, but none explains all the reported effects. Most studies of the effects of AEDs on bone tissue are cross-sectional. There are only a few longitudinal studies, and there are limited data regarding the newer AEDs (ref. ${ }^{6}$ ).

Lacosamide (LCM) (SPM 927, formerly harkoseride), the R-enantiomer of 2-acetamido- $N$-benzyl-3-methoxypropionamide, is a chemical compound with anticonvulsant and anti-nociceptive properties. In November 2007, a new drug application was filed with the FDA for use of LCM as adjunctive therapy in the treatment of partial-onset seizures in adults with epilepsy. LCM was approved in Europe on September 3, 2008 as adjunctive therapy in the treatment of partial-onset seizures, with or without secondary generalization, for patients with epilepsy of 16 years or older ${ }^{7}$. Sex hormone deficiency increases the risk of developing antiepileptic drug-induced osteopathy (AEDs-O) (ref. ${ }^{8}$ ).

We report here our findings on the impact of LCM on bone mineral density (BMD), bone mineral content (BMC), bone metabolism markers, and bone biomechanical properties in orchidectomised (ORX) rats fed on LCMenriched diet for 12 weeks. Apart from this study, to our knowledge there are no other full-text studies evaluating the effect of LCM on bone tissue. We have found one study referring to the absence of changes in BMD in juvenile dogs that has been published only in the form of an abstract ${ }^{9}$. 


\section{METHODS}

\section{Animals}

All animals received humane care in accordance with the guidelines set by the institutional Animal Use and Care Committee of Charles University in Prague, Faculty of Medicine in Hradec Kralove, Czech Republic. The protocol of the experiment was approved by the same committee. The experiments used eight-week-old male albino Wistar rats (Biotest s.r.o., Konarovice, Czech Republic). The animals were housed in groups of 4 in plastic cages. During the experimental period the animals were maintained under controlled conventional conditions $(12 \mathrm{~h}$ light and $12 \mathrm{~h}$ dark, temperature $22 \pm 2{ }^{\circ} \mathrm{C}$, air humidity 30 $70 \%$ ). Tap water, standard laboratory diet (SLD, VELAS, a.s., Lysa nad Labem, Czech Republic) and SLD enriched with LCM were given ad libitum. The weight of the rats was monitored once a week.

\section{Experiment}

Rats weighing $(270 \pm 7 \mathrm{~g})$ at the beginning of the experiment were divided into two groups of 8 animals:

1. CON-ORX: orchidectomised control fed with SLD

2. LCM-ORX: orchidectomised rat fed with SLD enriched with LCM (18 mg/25 g of the diet; Lacosamid, UCB Pharma)

At the beginning of the experiment the rats $(\mathrm{CON}$ ORX and LCM-ORX) underwent bilateral orchidectomy under ether anaesthesia. On the second day after operation the LCM-ORX began to receive SLD enriched with LCM and the CON-ORX only SLD, both diets ad libitum. After 12 weeks, the animals were sacrificed by exsanguination from the abdominal aorta under ether anesthesia, and the obtained serum was aliquoted and stored at $-80{ }^{\circ} \mathrm{C}$ for ensuing biochemical analyses. Both tibiae and femurs of the sacrificed rats were dissected free of soft tissue, wrapped in gauze moistened with saline and frozen to $-80^{\circ} \mathrm{C}$ till the time of analysis.

\section{Analysis of serum and bone homogenates}

In the blood serum, the levels of osteoprotegerin (OPG) and insulin-like growth factor 1 (IGF-1) were determined by the ELISA (Enzyme-Linked Immunosorbent Assay) method. The blood serum levels of LCM were determined in the middle and at the end of the experiment. LCM was assayed by modified high-performance liquid chromatography with diode array detection ${ }^{10}$. Sample preparation included precipitation of plasma proteins: $200 \mu \mathrm{L}$ of acetonitrile and $20 \mu \mathrm{L}$ of zinc sulphate solution ( $10 \%$ ) were added to $100 \mu \mathrm{L}$ of plasma samples in $1.5-\mathrm{mL}$ polypropylene centrifugation tubes. The tubes were vortexed for 120 seconds and centrifuged at $15,000 \mathrm{rpm}$ for 10 minutes. The supernatant $(30 \mu \mathrm{L})$ was injected into the HPLC system. Analysis was performed on a 2695 Waters Separations Module equipped with 996 photodiode array detector and Peltier columnthermostat Jet-Stream (Thermotechnic Products). Data acquisition and processing were provided with Empower Software (Waters). The analytical column was Zorbax SB-C8 (Agilent Technologies) - 150 x 4.6 mm, $3.5 \mu \mathrm{m}$.
The analytical precolumn was Symmetry C18 Guard Column - 20 × $3.9 \mathrm{~mm}, 5 \mu \mathrm{m}$ (Waters). The mobile phase was pumped at flow rate $0.8 \mathrm{~mL} / \mathrm{min}$ and consisted of acetonitrile:formic acid $0.1 \%(30: 70, \mathrm{v} / \mathrm{v})$. Temperature on the column was set at $30^{\circ} \mathrm{C}$, and injection volume was $30 \mu \mathrm{L}$. LCM concentration was determined at a wavelength of $215 \mathrm{~nm}\left(\right.$ ref. $\left.^{10}\right)$.

In bone homogenate, the levels of the markers carboxy-terminal cross-linking telopeptide of type I collagen (CTX-I), aminoterminal propeptide of procollagen type I (PINP), bone alkaline phosphatase (BALP) and bone morphogenetic protein 2 (BMP-2) were determined also using the ELISA method. The homogenate was prepared from the tibia. After animal sacrifice, both tibiae were carefully excised; after removal of all the surrounding skin, muscle and other soft tissue, they were stored at $-80{ }^{\circ} \mathrm{C}$ until required. The proximal part of the tibia $(0.1 \mathrm{~g})$ was disrupted and homogenized in $1.5 \mathrm{~mL}$ of phosphate buffer (PBS, PAA Laboratories GmbH, Pasching, Austria) with MagNA Lyser instrument (Roche Applied Science, Germany) at $6500 \mathrm{rpm}$ for $20 \mathrm{~s}$ and cooled on the MagNA Lyser Cooling Block. This procedure was repeated three times.

The tissue homogenate was centrifuged at 10,000 $\mathrm{g}$ at $4{ }^{\circ} \mathrm{C}$ for $10 \mathrm{~min}$. The supernatant was obtained and stored at $-80{ }^{\circ} \mathrm{C}$. Determination of the levels of bone markers was carried out using kits from Uscn Life Science Inc., Wuhan, China (PINP, Procollagen I N-Terminal Propeptide, pg/mL; OPG, Osteoprotegerin, pg/mL; IGF1 Insulin Like Growth Factor 1, pg/mL; CTX-I, Cross Linked C-Telopeptide Of Type I Collagen; pg/mL; BALP, Alkaline Phosphatase, Liver/Bone/Kidney, ng/mL; BMP2, Bone Morphogenetic Protein 2, pg/mL).

\section{Dual energy X-ray absorptiometry analysis}

The rat bone mineral density (BMD, $\mathrm{g} / \mathrm{cm}^{2}$ ) was measured by means of dual energy X-ray absorptiometry (DEXA) on a Hologic Delphi A device (Hologic, MA, USA) at the Osteocentre of the Faculty Hospital Hradec Kralove, Czech Republic. Before measurements, a tissue calibration scan was performed with the Hologic phantom for the small animal. Bone mineral density of the whole body, in the area of the lumbar vertebrae and in the area of the femur was evaluated by computer using the appropriate software programme for small animals (DEXA; QDR-4500A Elite; Hologic, Waltham, MA, USA). All animals were scanned by the same operator.

\section{Biomechanical testing procedure}

Mechanical testing of the rat femoral shaft and femoral neck was done with a special electromechanical custom-made testing machine (Martin Kosek \& Pavel Trnecka, Hradec Kralove, Czech Republic) as described in a previous report ${ }^{11}$. For the three-point bending test, the femur was supported in the anteroposterior direction on a holding device, with the two support points $18 \mathrm{~mm}$ apart. A small stabilizing preload to $10 \mathrm{~N}$ was used to fix the bone between the contacts. A constant deformation rate of $6 \mathrm{~mm} / \mathrm{min}$ as applied until maximal load failure and breaking strength (maximum load, N) 
were recorded. Once the bone was broken, the thickness of the cortical part of the bone was measured by means of a sliding micrometer (OXFORD 0-25MM 30DEG POINTED MICROMETER, Victoria Works, Leicester, Great Britain). The proximal part of the femur was used for compression test of the femoral neck. The diaphysis of the bone was embedded into a container using a methacrylate resin and a vertical load was applied to the top of the femoral head. A small stabilizing preload to $10 \mathrm{~N}$ was applied and then advanced at a constant speed of $6 \mathrm{~mm} / \mathrm{min}$ until failure of the femoral neck. The breaking strength (maximum load, $N$ ) was recorded by the measuring unit (Digitalanzeiger 9180, Burster praezisiosmesstechnik Gmbh \& co kg, Gernsbach, Germany). All bones were analyzed by the same operator.

\section{Analysis}

Data were analyzed using Statistica v.10 (USA) software. Because the Shapiro-Wilk $W$-test for normality of data indicated that normal distribution was unlikely, nonparametric tests were used in subsequent analyses. Results were expressed in the form of medians, as well as lower and upper quartiles. Data of CON-ORX and LCM-ORX groups were compared using the Mann-Whitney U test to assess differences. Statistical significance was indicated by $P$-values $<0.05$ in all calculations.

\section{RESULTS}

Comparison of body composition showed a significantly lower fat mass in total $(16.5 \mathrm{~g})$ as well as in relative $(4.1 \%)$ expression in those rats supplied with LCM. The contrast in fat expressed as a percentage was $18.3 \%$ (100* $\left(1-\mathrm{fat}_{\mathrm{LCMR}} / \mathrm{fat}_{\mathrm{CONR}}\right)$ between the groups. Also, the weight of rats in the CON-ORX groups was found to be higher ( $37 \mathrm{~g}$ ) compared to the LMC-ORX group, but this difference was not statistically significant - for details see Table 1.

Among the tested bone markers only aminoterminal propeptide of procollagen type I was significantly lower in the LCM-ORX group, by $29.6 \mathrm{pg} / \mathrm{L}$, representing an intergroup contrast of $37.4 \%$. Particular values and a detailed list of the other followed parameters are displayed in Table 2.

The mineral density of bone evaluated in the whole body and in the area of the lumbar vertebrae did not show any significant differences between the groups; however in the area of the left as well as the right femur we found significantly lower density (left $0.18 \mathrm{~g} / \mathrm{cm}^{2}$ and right $0.17 \mathrm{~g} / \mathrm{cm}^{2}$ ) in the LCM-ORX group compared to the control group (left BMD $0.20 \mathrm{~g} / \mathrm{cm}^{2}$ and right BMD $0.20 \mathrm{~g} / \mathrm{cm}^{2}$ ). The contrast between groups was $8.8 \%$ in the left BMD and $12.0 \%$ in the right BMD. The mineral content did not differ statistically between the groups. The listed values are medians; for interquartile intervals and other significant parameters see Table 3 .

Table 1. Body weight and fat mass.

\begin{tabular}{|c|c|c|c|}
\hline Parameter & $\begin{array}{c}\text { CON-ORX }(n=8) \\
{[25 \%-75 \%]}\end{array}$ & $\begin{array}{c}\text { LCM-ORX }(n=8) \\
{[25 \%-75 \%]}\end{array}$ & $\begin{array}{c}\text { Man-Whitney U Test } \\
{[P]}\end{array}$ \\
\hline Weight (g) & $523[489-543]$ & 486 [471 - 499] & 0.189 \\
\hline Fat $(\mathrm{g})$ & $90.3[83.1-127.2]$ & $73.8[65.1-85.2]$ & 0.024 \\
\hline Fat $(\%)$ & $22.1[20.1-26.7]$ & $18.0[16.1-20.0]$ & 0.041 \\
\hline Lean body mass (g) & $326.2(310.9-378.8)$ & $340.9(326.9-350.9)$ & 0.495 \\
\hline
\end{tabular}

Data are expressed as median $\left(25^{\text {th }}-75^{\text {th }}\right.$ percentiles $)$

Table 2. Levels of bone markers.

\begin{tabular}{|c|c|c|c|}
\hline Parameter & $\begin{array}{c}\text { CON-ORX }(n=8) \\
{[25 \%-75 \%]}\end{array}$ & $\begin{array}{c}\text { LCM-ORX }(n=8) \\
{[25 \%-75 \%]}\end{array}$ & $\begin{array}{c}\text { Man-Whitney U Test } \\
{[P]}\end{array}$ \\
\hline OPG (pg/mL) & $54.7[51.9-58.5]$ & $57.9[51.1-54.5]$ & 0.372 \\
\hline IGF-1 (pg/mL) & $1.11[1.06-1.21]$ & 1.17 [1.05 - 1.29] & 0.564 \\
\hline CTX-1 (pg/mL) & $0.97[0.76-1.57]$ & $0.90[0.64-1.63]$ & 0.772 \\
\hline PINP (pg/mL) & $79.0[71.9-81.5]$ & $49.4[34.5-67.6]$ & 0.005 \\
\hline BALP (ng/mL) & $1.16[0.76-1.46]$ & $0.88[0.70-1.14]$ & 0.495 \\
\hline BMP-2 (pg/mL) & 876 [610 - 1010] & 824 [763 - 1055] & 0.958 \\
\hline
\end{tabular}

OPG, osteoprotegerin; IGF-1, insulin-like growth factor 1; CTX-I, carboxy-terminal cross-linking telopeptide of type I collagen; PINP, aminoterminal propeptide of procollagen type I; BALP, bone alkaline phosphatase; BMP-2, bone morphogenetic protein 2.

Data are expressed as median $\left(25^{\text {th }}-75^{\text {th }}\right.$ percentiles $)$ 
Table 3. Comparison of values from DXA analysis and values of biomechanical testing.

\begin{tabular}{lccc}
\hline & CON-ORX $(\mathrm{n}=8)$ & LCM-ORX $(\mathrm{n}=8)$ & $\begin{array}{c}\text { Man-Whitney } \\
\text { U Test }[P]\end{array}$ \\
\hline DEXA & {$[25 \%-75 \%]$} & {$[25 \%-75 \%]$} & 0.066 \\
Whole body BMD, g/cm ${ }^{2}$ & & & 0.318 \\
W BMC (g) & $0.175[0.171-0.176]$ & $0.169[0.165-0.171]$ & 0.004 \\
LF BMD, g/cm ${ }^{2}$ & $14.0[13.3-14 ., 4]$ & $13.5[13.1-13.9]$ & 0.793 \\
LF BMC (g) & $0.197[0.192-0.199]$ & $0.179[0.169-0.185]$ & 0.001 \\
RF BMD, g/cm ${ }^{2}$ & $0.276[0.258-0.290]$ & $0.266[0.259-0.279]$ & 0.564 \\
RF BMC (g) & $0.198[0.188-0.211]$ & $0.175[0.160-0.179]$ & 0.793 \\
Lumbar columna (L3-L5) BMD (g/cm $\left.{ }^{2}\right)$ & $0.259[0.236-0.280]$ & $0.259[0.248-0.284]$ & 0.495 \\
Lumbar columna (L3-L5) BMC (g) & $0.214[0.210-0.223]$ & $0.212[0.207-0.223]$ & 0.495 \\
\hline Biomechanical testing measurement & $0.571[0.546-0.598]$ & $0.575[0.542-0.614]$ & 0.958 \\
LF length, mm & & & 0.958 \\
RF length, mm & $37.5[36.6-38.0]$ & $37.3[36.9-38.6]$ & 0.753 \\
LF diameter, mm & $37.4[36.6-37.8]$ & $37.4[37.2-37.8]$ & 0.263 \\
RF diameter, mm & $3.63[3.51-3.95]$ & $3.64[3.62-3 ., 67]$ & 0.958 \\
Cortical LF thickness, mm & $3.63[3.53-3.72]$ & $3.65[3.47-3.95]$ & 1.000 \\
Cortical RF thickness, mm & $0.720[0.665-0.740]$ & $0.705[0.695-0.750]$ & 0.564 \\
Maximal load of the left femoral shaft, N & $0.705[0.665-0.735]$ & $0.695[0.680-0.740]$ & 0.897 \\
Maximal load of the right femoral shaft, N & $214[197-236]$ & $218[207-229]$ & 0.355 \\
Maximal load of left femoral neck, N & $216[203-230]$ & $224[204-235]$ & $146[128-163]$ \\
Maximal load of right femoral neck, N & $144[135-152]$ & $149[141-161]$ & \\
\hline
\end{tabular}

LF- left femur, RF - right femur, BMD - bone mineral density, BMC - bone mineral content. Data are expressed as median $\left(25^{\text {th }}-75^{\text {th }}\right.$ percentiles $)$ CON-ORX, orchidectomised control fed with standard laboratory diet; LCM-ORX, orchidectomised rat fed with standard laboratory diet enriched with lacosamide.

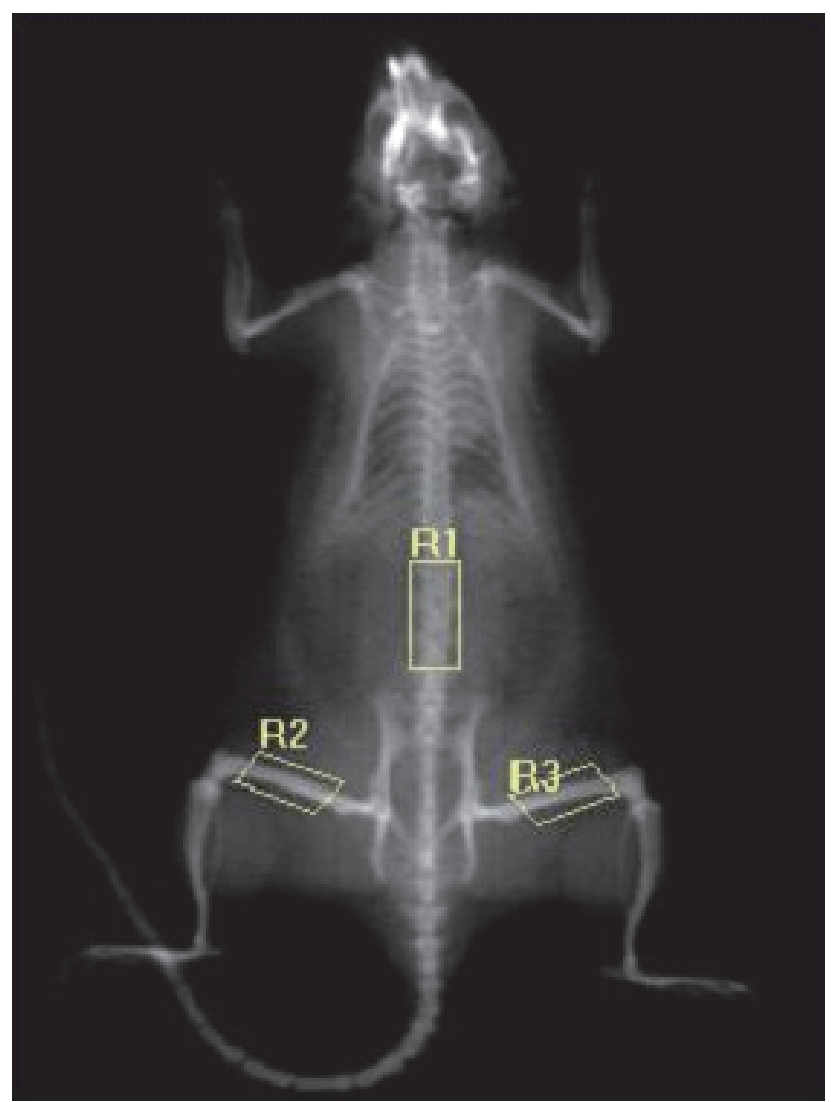

Fig. 1. Evaluation of BMD in three areas of the rat skeleton. R1 - lumbar columna (L3-L5);

R2 - left femur;

R3 - right femur
The Mann-Whitney $U$ test also showed that medians of biomechanical and geometric parameters of right and left femurs did not differ (see Table 3).

The level of lacosamide in LCM-ORX at the end of the experiment: $13.49 \mathrm{umol} / \mathrm{L}(12.96$ - 14.59).

\section{DISCUSSION}

The aim of this study was to evaluate the effect of LCM on BMD, bone markers, and biomechanical quality using the mature ORX rat model. The effect of LCM on bone tissue has not yet been investigated, except for studies Cornet et al 2010 in juvenile dogs (published only in the form of an abstract) (ref. ${ }^{9}$ ). In this study, ORX rats fed with SLD enriched with LCM (LCM-ORX) had significant loss of BMD at the left and right femur after 12 weeks when compared to the control (CON-ORX). However, no significant differences in biomechanical and geometric parameters of rat right and left femurs were observed. Evaluation of bone turnover using biochemical markers specific for both bone formation (BALP, BMP-2, PINP, OPG, IGF-1) and bone resorption (CTX-I) was without significant difference with the exception of PINP.

With respect to AEDs, phenytoin (PHT), phenobarbital (PB), and primidone (PRM) are most consistently associated with a negative impact on bone. Carbamazepine (CBZ) and valproate (VPA) may also result in bone abnormalities, but the data are mixed. Current studies suggest that lamotrigine (LTG) has limited (if any) effect, 
but again, the data are inconsistent. Other AEDs have received limited study ${ }^{12}$ but there is increasing evidence supporting the notion that topiramate (TPM) may have negative impact on bone health ${ }^{13-15}$.

The significance of bone turnover markers for diagnosis of antiepileptic drug-induced osteopathy (AEDs-O) is controversial: although PHT is well-known to cause significant loss of BMD and BMC, only modest changes in markers of bone turnover have been observed in animals ${ }^{16,17}$. Similarly, in a longitudinal study of premenopausal women treated with PHT, bone turnover markers remained unchanged after 1 year, except for a significant decline in urine N-telopeptide. This result is unclear and difficult to explain, particularly in view of the significant observed femoral neck bone loss ${ }^{18}$. Conflicting data exist regarding the effects of $\mathrm{CBZ}$ on $\mathrm{BMD}$ and bone turnover $^{8,19-21}$. VPA in animals reduced BMD and BMC and increased bone turnover ${ }^{16}$. There are mixed data in humans. Some have observed that VPA monotherapy decreased BMD and increased both markers of bone formation and resorption significantly ${ }^{22,23}$, but in the longitudinal study of young women mentioned above, the BMD was stable and bone turnover markers remained unchanged after 1 year of VPA treatment ${ }^{18}$. There are scarce data regarding LTG. So far LTG has not been shown to cause significant effects on BMD and bone turnover ${ }^{18,21}$, except significantly increased osteocalcin, a marker of bone formation referred to in one study ${ }^{24}$.

In our study we noticed a significant change in the PINP. PINP is a marker of bone formation, which was significantly lower in LCM-ORX. To our knowledge, there have appeared no studies with AEDs (or with CYP2C19 inhibitors) in which changes in the level of PINP were monitored. Some further research will be necessary to verify the role and the importance of PINP in the diagnosis, and more precisely in the pathophysiology of AEDs-O. We discovered only one prospective study testing the influence of LCM on BMD in gonadally intact subjects, in which the authors claim an absence of influence of LCM on BMD (ref. ${ }^{9}$ ). However, we monitored the significant decline of BMD at the left and right femur. We assume then that long-lasting exposure to LCM can represent a certain risk to the health of bone in the setting of the gonadal insufficiency. It is complicated to determine how high the risk will be in comparison to the other AEDs. In the case of levetiracetam (LEV) we monitored a significant decline of BMD on the same model in only 1 out of 4 locations of measurement (left femur), and moreover, we did not record significant changes in the mechanical durability of the bone ${ }^{25}$. In the case of LTG and TPM, we recorded on the same model both significant decline of BMD (at 3 measurement locations out of 4) and significant reduction in the mechanical durability of the bone (supplied data). The old AEDs have not been tested on the ORX rat model so far. However, in models of gonadally intact rats, significant reduction of BMD after using the old AEDs has been observed (see text above).

The mechanism of the effect of LCM on bone is unclear. LCM has been shown to produce a significant effect in rodents consistent with anxiolysis: LCM increased the suppression ratio in a conditioned emotional response test, and reduced the number of marbles buried in the marble burying assay ${ }^{26}$. In rodents, physical activity prevents decrease in BMD as it does in humans, which suggests that increased physical activity could be useful in the prevention of bone mineral loss, regardless of gonadal hormone deficiency ${ }^{27}$. Therefore reduced locomotor activity could be the factor contributing to significant decrease LF-BMD and RF-BMD in LCM-ORX compared to CON-ORX.

In our study, LCM-ORX had significantly lower fat mass compared with CON-ORX. LCM showed no potential to induce or to inhibit cytochrome $\mathrm{P} 450$ isoforms except for CYP2C19 (60\% inhibition) (ref. ${ }^{28}$ ). CYP2C19 is one of the (most) important isoforms involved in the metabolism of sex hormones. CYP2C19 catalyzes the $17 \beta$-hydroxy dehydrogenation and $16 \alpha$ hydroxylation of estradiol, and 17 $\beta$-hydroxy dehydrogenation is the main metabolism pathway at low estradiol concentrations ${ }^{29,30}$. The main pathway of testosterone oxidative metabolism by human liver microsomes is the formation of $1 \beta-, 2 \alpha-$ $/ \beta$-, $6 \beta$-, 15 $\beta$-, and 16 $\beta$-hydroxytestosterones, mainly catalyzed by CYP2C9, CYP2C19, and CYP3A4 (ref. ${ }^{31}$ ). In a study in the ORX rat model with different doses of testosterone replacement there were no significant differences in fat mass ${ }^{32}$, and in another study, 17 $\beta$-estradiol prevented the ORX-related increase of fat mass, whereas $5 \alpha$-dihydrotestosterone did not ${ }^{33}$. Thus we propose that inhibition of estrogen metabolism may be the cause of lower fat mass in LCM-ORX rather than inhibition of androgen metabolism.

There are several limitations that should be considered in evaluating the present study. Firstly, the three-month follow-up period may be too short for monitoring changes in biomechanical properties of the bone tissue. Secondly, the sample size was small: although statistical significance is evident, the capacity to identify possible variables of confusion is limited. Finally, behavioral activity was neither controlled nor assessed.

\section{CONCLUSION}

In summary, in this study, LCM-ORX had significant loss of BMD at the left and right femur and significant decline in PINP compared to the control. It will be necessary to carry out further studies to validate the findings of this study and to elucidate the exact mechanism of the significant loss of femur BMD. Further studies are warranted to establish whether LCM has a clinically significant effect on BMD exclusively in the model of gonadectomized rats, or whether the effect applies also in the model of gonadally intact animals, and in the respective human models.

Despite the above-mentioned limitations, this study contributes significantly to our knowledge about the effect of LCM on bone tissue. 


\section{ACKNOWLEDGEMENT}

This study was supported by a Research Project of PRVOUK 37/11 Charles University in Prague project, SVV-2011-262902, SVV -2012 - 264902 and MH CZ DRO (UHHK, 00179906). The authors wish to thank Dagmar Jezkova and Katerina Sildbergerova for their skilful technical assistance throughout the experiment. The authors are grateful to Ian McColl MD, PhD for assistance with the manuscript.

Author contributions: JS, SF, JM: literature search, manuscript writing; JS, PZ, VP: study design; SF: figures; SF, HZ, JM: data collection; JS, JK, JH, VP, PZ: data interpretation; JS, JK, JH: statistical analysis.

Conflict of interest statement: None declared.

\section{REFERENCES}

1. Savica R, Beghi E, Mazzaglia G, Innocenti F, Brignoli O, Cricelli C Caputi AP, Musolino R, Spina E, Trifirň G. Prescribing patterns of antiepileptic drugs in Italy: nationwide population-based study in the years 2000-2005. Eur J Neurol 2007;14(12):1317-21.

2. Johannessen LC, Larsson PG, Rytter E, Johannessen SI. Antiepileptic drugs in epilepsy and other disorders-A population-based study of prescriptions. Epilepsy Res 2009;87(1):31-9.

3. Van de Vrie-Hoekstra NW, de Vries TW, van den Berg PB, Brouwer OF, de Jong-van den Berg LT. Antiepileptic drug utilization in children from 1997-2005 a study from the Netherlands. Eur J Clin Pharmaco 2008;64(10):1013-20.

4. Schmid F. Osteopathien bei antiepileptischer D auerbehandlung Fortschr Med 1967;85:381-2.

5. Kruse R. Osteopathien bei antioepileptischer Langzeittherapie (vorläufige Mitteilung). Mschr Kinderheilkd 1968;116:378-80.

6. Pack A. Bone health in people with epilepsy: is it impaired and what are the risk factors? Seizure 2008;17(2):181-6.

7. Halford JJ, Lapointe M. Clinical Perspectives on Lacosamide. Epilepsy Currents 2009;9(1):1-9.

8. Carbone LD, Johnson KC, Robbins J, Larson JC, Curb JD, Watson K Gass M, Lacroix AZ. Antiepileptic drug use, falls, fractures, and BMD in postmenopausal women: findings from the women's healthinitiative (WHI). J Bone Minereal res 2010;25(4):873-81.

9. M. Cornet, D. Tytgat and M. Léonard. Lacosamide does not alter bone densitometry parameters in juvenile dogs. Abst. American Epilepsy Society 2010; Abstract No.2.191.

10. Greenaway C, Ratnaraj N, Sander JW, Patsalos PN. A Highperformance liquid chromatography assay to monitor the new antiepileptic drug lacosamide in patients with epilepsy. Ther Drug Monit 2010;32(4):448-52.

11. Gradosova I, Zivna H, Svejkovska K, Palicka V, Tichy M, Zivny P. The role of atorvastatin in bone metabolism in male albino Wistar rats. Pharmazie 2011;66:606-10.

12. Pack AM. Treatment of epilepsy to optimize bone health. Curr Treat Options Neurol 2011;13(4):346-54.

13. Coppola G, Fortunato D, Auricchio G, Mainolfi C, Operto FF, Signoriello G, Pascotto A, Salvatore M. Bone mineral density in children, adolescents, and young adults with epilepsy. Epilepsia 2009;50(9):2140-6.

14. Heo K, Rhee Y, Lee HW, Lee SA, Shin DJ, Kim WJ, Song HK, Song K, Lee $\mathrm{BI}$. The effect of topiramate on bone mineral density and markers of bone mineral metabolism in premenopausal women with epilepsy. Epilepsia 2011;52(10):1884-9.

15. Ali II, Herial NA, Orris M, Horrigan T, Tietjen GE. Migraine prophylaxis with topiramate and bone health in women. Headache 2011;51(4):613-6.

16. Nissen-Meyer LS, Svalheim S, Taubøll E, Reppe S, Lekva T, Solberg LB, Melhus G, Reinholt FP, Gjerstad L, Jemtland R. Levetiracetam, phenytoin, and valproate act differently on rat bone mass, structure, and metabolism. Epilepsia 2007;48(10):1850-60.

17. Moro-Alvarez MJ, Díaz Curiel M, de la Piedra C, Marińoso ML, Carrascal MT. Bone disease induced by phenytoin therapy: clinical and experimental study. Eur Neurol 2009;62(4):219-30.

18. Pack AM, Morrell MJ, Randall A, McMahon DJ, Shane E. Bone health in young women with epilepsy after one year of antiepileptic drug monotherapy. Neurology 2008;70(18):1586-93.

19. Valimaki MJ, Tiihonen M, Laitinen K. Bone mineral density measured by dual-energy x-ray absorptiometry and novel markers of bone formation and resorption in patients on antiepileptic drugs. J Bone Miner Res 1974;9(5):631-7.

20. Verrotti A, Greco R, Latini G, Morgese G, Chiarelli F. Increased bone turnover in prepubertal, pubertal, and postpubertal patients receiving carbamazepine. Epilepsia 2002;43(12):1488-92.

21. Sheth RD, Hermann BP. Bone mineral density with lamotrigine monotherapy for epilepsy. Pediatr Neurol 2007;37(4):250-4.

22. Sato Y, Kondo I, Ishida S, Motooka H, Takayama K, Tomita Y, Maeda $\mathrm{H}$, Satoh K. Decreased bone mass and increased bone turnover with valproate therapy in adults with epilepsy. Neurology 2001;57(3):445-9.

23. Verrotti A, Agostinelli S, Coppola G, Parisi P, Chiarelli F. A 12-month longitudinal study of calcium metabolism and bone turnover during valproate monotherapy. Eur J Neurol 2010;17(2):232-7.

24. Kim SH, Lee JW, Choi KG, Chung HW, Lee HW. A 6-month longitudina study of bone mineral density with antiepileptic drug monotherapy. Epilepsy Behav 2007;10(2):291-5.

25. Fekete S, Simko J, Gradosova I, Malakova J, Zivna H, Palicka V, Zivny $P$. The effect of levetiracetam on rat bone mass, structure and metabolism. Epilepsy research 2013;107(1-2):56-60.

26. Higgins GA, Breysse N, Undzys E, Kuo C, Joharchi N, Derksen DR, Xin T, Isaac M, Slassi M. The anti-epileptic drug lacosamide (Vimpat) has anxiolytic property in rodents. Eur J Pharmacol 2009;624(1-3):1-9.

27. Horcajada-Molteni MN, Davicco MJ, Coxam V, Lebecque $P$, Dominguez B, Ritz P, Culioli J, Barlet JP. Treadmill running starting 3 months after orchidectomy restores femoral bone mass in rats. Eur J Appl Physiol Occup Physiol 1999;79(3):251-9.

28. Beyreuther BK, Freitag J, Heers C, Krebsfänger N, Scharfenecker U, Stöhr T. Lacosamide: A Review of Preclinical Properties. CNS Drug Rev 2007;13(1):21-42

29. Cribb AE, Knight MJ, Dryer D, Guernsey J, Hender K, Tesch M. Role of polymorphic human cytochrome P450 enzymes in estrone oxidation. Cancer Epidemiol Biomarkers Prev 2006;15:551-8.

30. Cheng ZN, Shu Y, Liu ZQ, Wang LS, Ou-Yang DS, Zhou HH. Role of cytochrome P450 in estradiol metabolism in vitro. Acta Pharmacol Sin 2001;22:148-54.

31. Man Ho Choi, Paul L. Skipper, John S. Wishnok, Steven R. Tannenbaum: Characterization of testosterone $11 \beta$-hydroxylation catalyzed by human liver microsomal cytochromes P450. Drug metabolism and disposition 2005;33:714-8.

32. Vanderschueren $D$, Vandenput $L$, Boonen S, Van Herck $E$, Swinnen JV, Bouillon R. An aged rat model of partial androgen deficiency: prevention of both loss of bone and lean body mass by low-dose androgen replacement. Endocrinology 2000;141(5):1642-7.

33. Vandenput L, Boonen S, Van Herck E, Swinnen JV, Bouillon R, Vanderschueren $\mathrm{D}$. Evidence from the aged orchidectomized male rat model that 17 beta-estradiol is a more effective bone-sparing and anabolic agent than 5alpha-dihydrotestosterone. J Bone Miner Res 2002;7(11):2080-6. 